\author{
H. R. Hamidzadeh \\ N. N. Sawaya \\ Mechanical Engineering \\ Department \\ South Dakota State University \\ Brookings, SD 57007
}

\section{Free Vibration of Thick Multilayer Cylinders}

In this study of the free vibration of multilayer thick cylinders, the medium is modeled by laminated linear viscoelastic cylinders of an infinite extent. The analytical modeling is based on three-dimensional wave propagation utilizing constant complex elastic moduli. The solution is achieved by determining the displacements and stresses for each interface and by complying with requirements at the interfaces. A propagator matrix relating the boundary displacements to boundary stresses is developed. Dimensionless natural frequencies and modal loss factors for different circumferential and axial wave numbers are determined. The validity of the proposed method is verified by comparing the results for one-, two-, and three-layer elastic cylinders with properties similar to those reported for an equivalent single layer. (c) 1995 John Wiley \& Sons, Inc.

\section{INTRODUCTION}

Most studies of the vibrations of sandwich cylinders are based on the assumptions of thin shell theory for a significant number of thick cylindrical structures. The assumptions used in shell theory for cylindrical structures may not always be valid. In spite of this situation, few studies have been addressed to the analysis of the free vibrations of thick cylindrical structures.

Armenakas et al. (1969) analyzed free vibration of thick elastic cylinders and presented tables of dimensionless natural frequencies for a wide range of geometrical possibilities. Armenakas (1967) investigated the propagation of harmonic waves with arbitrary circumferential nodes traveling in traction free composite cylindrical shells. The composite shells consisted of an inner layer composed of one material bounded by and bonded to an outer casing made of a different elastic material. Armenakas (1971) studied the effect of changes in shell parameters on the frequencies and mode shapes for the first few modes. Huang and Dong (1984) utilized a stiffness method to consider wave propagation in laminated aniso- tropic cylinders with an arbitrary number of lamina. Braga and coworkers (1990) studied harmonic wave propagation in a fluid-loaded cylinder composed of isotropic elastic layers. In their computation, they developed an analytical method to determine impedance of the laminated shell. Hamidzadeh and Chandler (1991) investigated circumferential harmonic vibrations of viscoelastic thick cylinders of infinite extent by employing the two-dimensional elastodynamic theory. Rattanawangcharoen and Shah (1991) studied the propagation of harmonic waves in laminated isotropic elastic cylinders. They provided dimensionless natural frequencies of a two-layer cylinder for a circumferential wave number and a range of axial wave numbers. Rattanawangcharoen et al. (1992) also analyzed the effects of the circumferential wave number, ply lay up configuration, number of layers, and thickness-to-radius ratio on the dispersion characteristics.

This article addresses the study of free vibration of multilayer viscoelastic cylinders. To introduce viscoelastic damping in the composite cylinder, the analysis allows complex shear modulus for each layer. The problem is formulated in the

Received April 28, 1994; Accepted May 8, 1995.

Shock and Vibration, Vol. 2, No. 5, pp. 393-401 (1995)

(C) 1995 by John Wiley \& Sons, Inc.

CCC 1070-9622/95/050393-09 
form of matrix equations that relates displacements of a point in the medium to the boundary stresses. An analytical approach for a single frequency independent viscoelastic layer is developed to obtain the displacements and stresses in terms of boundary stresses for any given circumferential and axial wave numbers. This formulation is used to develop a relation between displacements and stresses at the boundaries of each layer and consequently to obtain a propagator matrix relating displacement and stresses of the inner boundaries to the outer one. Invoking the zero boundary stresses leads to a frequency equation for free vibration of the multilayer viscoelastic cylinder. The analytical procedure described here is applicable to multilayer cylinders of arbitrary laminated configuration and any material damping for each layer.

\section{MODAL DISPLACEMENTS AND STRESSES FOR A CYLINDER}

The governing equations for an isotropic linearly viscoelastic cylinder in terms of displacement vector $\mathbf{u}$ and in the absence of prescribed body forces satisfy the following equation:

$$
(\lambda+\mu) \nabla \nabla \mathbf{u}+\mu \nabla^{2} \mathbf{u}=\rho \ddot{\mathbf{u}}
$$

where $\ddot{\mathbf{u}}=\partial^{2} \mathbf{u} / \partial \mathrm{t}^{2}, \lambda$ and $\mu$ are Lame's complex moduli and are presented by $\lambda=\lambda(1+i \beta)$ and $\mu=\mu(1+i \beta), \beta$ is the frequency independent material loss factor for the cylinder, and $\rho$ is the mass density of the body. The stress tensor $\sigma$ is related to the displacement vector by the following equation:

$$
\underline{\sigma}=\lambda \nabla \cdot \mathbf{u I}+\mu(\nabla \mathbf{u}+\mathbf{u} \nabla)
$$

where $\mathrm{I}$ is the unit tensor. As reported by Armenakas et al. (1969), the equilibrium equation, Eq. (1), can be solved by employing the potential function $\phi$ and $\mathbf{H}$ such that

$$
\mathbf{u}=\nabla \phi+\nabla \times \mathbf{H} .
$$

the potential functions are to satisfy the following wave equations:

$$
V_{1}^{2} \nabla^{2} \phi=\frac{\partial^{2} \phi}{\partial t^{2}}
$$

and

$$
V_{2}^{2} \nabla^{2} \mathbf{H}=\frac{\partial^{2} \mathbf{H}}{\partial t^{2}} .
$$

In the above equations, $V_{1}$ and $V_{2}$ are complex dilatational and shear wave velocities. From the foregoing equations, solutions to the potential functions for harmonic frequency of $\omega$ and axial and circumferential wave numbers $\zeta$ and $n$ are:

$$
\begin{aligned}
\phi & =f(r) \cos n \theta \cos (\omega t+\zeta z) \\
H_{r} & =g_{r}(r) \sin n \theta \cos (\omega t+\zeta z) \\
H_{\theta} & =g_{\theta}(r) \cos n \theta \cos (\omega t+\zeta z) \\
H_{z} & =g_{3}(r) \sin n \theta \cos (\omega t+\zeta z)
\end{aligned}
$$

where

$$
\begin{aligned}
f(r) & =C_{1} J_{n}(\alpha r)+C_{2} Y_{n}(\alpha r) \\
g_{3}(r) & =C_{3} J_{n}(\beta r)+C_{4} Y_{n}(\beta r) \\
2 g_{1}(r) & =g_{r}(r)-g_{\theta}(r) \\
& =2 C_{5} J_{n+1}(\beta r)+2 C_{6} Y_{n+1}(\beta r) \\
2 g_{2}(r) & =g_{r}(r)+g_{\theta}(r) \\
& =2 C_{7} J_{n-1}(\beta r)+2 C_{8} Y_{n-1}(\beta r)
\end{aligned}
$$

and

$$
\begin{aligned}
& \alpha^{2}=\frac{\omega^{2}}{V_{1}^{2}}-\zeta^{2} \\
& \beta^{2}=\frac{\omega^{2}}{V_{2}^{2}}-\zeta^{2} .
\end{aligned}
$$

It can be shown that one of the three potentials $g_{1}, g_{2}$, and $g_{3}$ can be set equal to zero, without loss of the generality of solution. Utilizing the property of the gauge invariance results in elimination of integration constants $C_{7}$ and $C_{8}$ in the above equations.

Using Eqs. (2) and (3) the modal displacements and stresses vector $\{\mathbf{R}\}$ can be written as:

$$
\{\mathbf{R}\}=[C S][S N]\{\mathbf{C}\}
$$

where

$$
\begin{aligned}
& \{\mathbf{R}\}=\left\{u_{r}, u_{\theta}, u_{z}, \sigma_{r r}, \sigma_{r \theta}, \sigma_{r z}\right\}^{T}, \\
& \{\mathbf{C}\}=\left\{C_{1}, C_{2}, C_{3}, C_{4}, C_{5}, C_{6}\right\}^{T}
\end{aligned}
$$

and $C_{i}$ are independent arbitrary constants. The elements of the $6 \times 6$ matrices $[C S]$ and $[S N]$ are given in the appendix. The elements of the matrix $[C S]$ are functions of frequency, time, coordinates $r, \theta$, and $Z$, as well as circumferential and 
axial wave numbers. The elements of the $[S N]$ matrix are functions of radius, frequency, material damping, material properties, and the abovementioned wave numbers.

\section{FORMULATION OF PROPAGATOR MATRIX}

The composite cylinder is assumed to be formed by $N$ viscoelastic layers bounded by inner and outer interfaces as shown in Fig. 1. The $i$ th layer $(i=1, N)$ has a thickness of $h_{i}=r_{i}-r_{i-1}$ and is bounded by inner and outer interfaces located at radii $r_{i-1}$ and $r_{i}$, respectively. Each of the viscoelastic layers is characterized by a complex dilatational wave velocity $V_{1 i}$, complex shear wave velocity $V_{2 i}$, density $\rho_{i}$, Poisson's ratio $\nu_{i}$, and material loss factor $\beta_{i}$. For the $i$ th layer, the model displacements and stresses vectors for inner and outer interfaces according to Eq. (16) can be written as:

$$
\begin{aligned}
\{\mathbf{R}\}_{i-1} & =[C S][S N]_{i-1}\{\mathbf{C}\} \\
\{\mathbf{R}\}_{i} & =[C S][S N]_{i}\{\mathbf{C}\} .
\end{aligned}
$$

Eliminating vector $\{\mathbf{C}\}$ in the above equations results in

$$
\{\mathbf{R}\}_{i}=[S N]_{i}[S N]_{i-1}^{-1}\{\mathbf{R}\}_{i-1}
$$

or

$$
\{\mathbf{R}\}_{i}=[P]_{i}\{\mathbf{R}\}_{i-1}
$$

where

$$
[P]_{i}=[S N]_{i}[S N]_{i-1}^{-1} .
$$

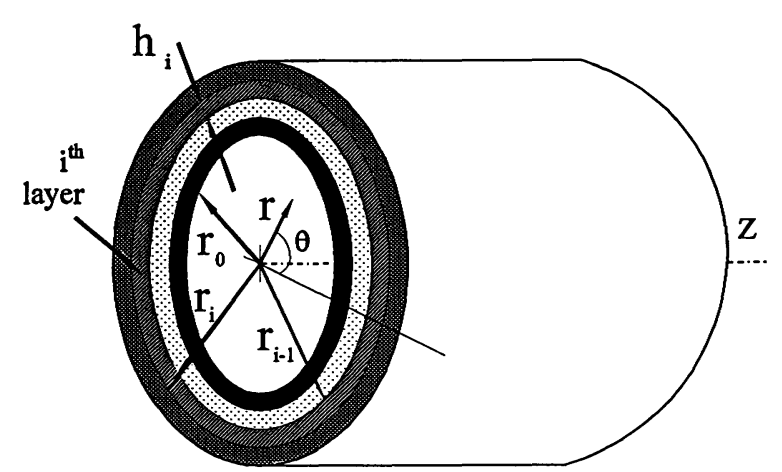

FIGURE 1 Cross section of multilayered circular cylinder.
Writing Eq. (18b) for all layers and eliminating $\{\mathbf{R}\}$ for all intermediate interfaces results in a relation between displacement and stress vectors at the inner and outer boundaries of the sandwiched cylinder.

$$
\{\mathbf{R}\}_{N}=[P]_{n}[P]_{n-1} \cdots[P]_{1}\{\mathbf{R}\}_{o}
$$

or

$$
\{\mathbf{R}\}_{N}=[Q]\{\mathbf{R}\}_{o}
$$

where

$$
[Q]=[P]_{n}[P]_{n-1}[P]_{n-2} \cdots[P]_{1} .
$$

Considering the traction free boundary conditions for free vibrations, Eq. (21) will be reduced to the following form:

$$
\left[\begin{array}{lll}
Q_{41} & Q_{42} & Q_{43} \\
Q_{51} & Q_{52} & Q_{53} \\
Q_{61} & Q_{62} & Q_{63}
\end{array}\right]\left\{\begin{array}{c}
u_{1} \\
v_{1} \\
w_{1}
\end{array}\right\}=\left\{\begin{array}{l}
0 \\
0 \\
0
\end{array}\right\}
$$

where $u_{1}, v_{1}$, and $w_{1}$ are amplitudes of radial, tangential, and axial components of displacement at any location in the inner surface of the cylinder, respectively.

Equating the determinant of the coefficient matrix in Eq. (22) to zero provides the frequency equation for the system. It should be indicated that the elements of the above matrix are all related to the circumferential and axial wave numbers, frequency, material loss factor, and thickness of each layer. Complex dimensionless natural frequencies $\left(\overline{\Omega_{j}}\right)$ for different modes can be computed using Muller's iterative method. The initial approximations are provided by sweeping the frequency with fine increments for the expected range. The dimensionless natural frequencies and the modal loss factors are extracted from the complex roots of the frequency equation by the following expressions:

$$
\begin{aligned}
\Omega_{j^{2}} & =\operatorname{Re}\left(\overline{\Omega_{j^{2}}}\right) \\
\eta_{j} & =\operatorname{Im}\left(\overline{\Omega_{j^{2}}}\right) / \operatorname{Re}\left(\overline{\Omega_{j^{2}}}\right) .
\end{aligned}
$$

\section{RESULTS FOR DIFFERENT CASE STUDIES}

To verify the validity of the developed procedure, several case studies were performed and the results are presented in the following. 


\section{Case 1: Elastic Cylinder}

Dimensionless natural frequencies for different circumferential wave numbers $(n=0,1,2,3$, and 4), ratios of $(H / L=0,0.1,0.5$, and 1$), H / R=$ 0.3 , and Poisson's ratio of 0.3 are computed and compared with those reported by Armenakas et al. (1969), where $H, R$, and $L$ are thickness, mean radius, and half wave length of the cylinder, respectively. These results are tabulated in Table 1 and comparison indicates excellent agreement.
Dimensionless natural frequencies are defined as $\Omega_{j}=\omega_{j} H /\left(\pi V_{2}\right)$, where $\omega_{j}$ is the $j$ th natural frequency and $V_{2}$ is the velocity of the shear wave for the cylinder.

\section{Case 2: Two-Layer Elastic Cylinder}

A two-layer elastic cylinder with the same mechanical properties is considered. Dimensionless natural frequencies for different circumferential wave numbers and two values for $H / L$ are com-

Table 1. Comparison of Dimensionless Natural Frequencies for Single Cylinder $(H / R=0.3)$

\begin{tabular}{|c|c|c|c|c|c|c|c|c|}
\hline \multirow[b]{2}{*}{$n$} & \multicolumn{8}{|c|}{ Dimensionless Natural Frequencies } \\
\hline & & $H / L$ & $\Omega_{1}$ & $\Omega_{2}$ & $\Omega_{3}$ & $\Omega_{4}$ & $\Omega_{5}$ & $\Omega_{6}$ \\
\hline \multirow[t]{8}{*}{0} & \multirow[t]{2}{*}{0.0} & Present & 0.00000 & 0.16305 & 1.00344 & 1.87870 & 2.00174 & 3.00116 \\
\hline & & AGH & 0.00000 & 0.16306 & 1.00344 & 1.87871 & 2.00174 & 3.00118 \\
\hline & \multirow[t]{2}{*}{0.1} & Present & 0.13828 & 0.18945 & 1.02108 & 1.85679 & 2.02643 & 3.00083 \\
\hline & & $\mathrm{AGH}$ & 0.13829 & 0.18946 & 1.02055 & 1.85681 & 2.03137 & 3.00076 \\
\hline & \multirow[t]{2}{*}{0.5} & Present & 0.31801 & 0.82790 & 1.33439 & 1.74402 & 2.33664 & 3.00068 \\
\hline & & $\mathrm{AGH}$ & 0.31790 & 0.82791 & 1.33441 & 1.74402 & 2.33665 & 3.00068 \\
\hline & \multirow[t]{2}{*}{1.0} & Present & 0.78333 & 1.42000 & 1.91154 & 2.00292 & 2.82861 & 3.07879 \\
\hline & & $\mathrm{AGH}$ & 0.78471 & 1.42002 & 1.91156 & 2.00294 & 2.82864 & 3.07880 \\
\hline \multirow[t]{8}{*}{1} & \multirow[t]{2}{*}{0.0} & Present & 0.00000 & 0.09583 & 0.22521 & 1.00819 & 1.03402 & 1.85844 \\
\hline & & $\mathrm{AGH}$ & 0.00000 & 0.09584 & 0.22524 & 1.00819 & 1.03404 & 1.85845 \\
\hline & \multirow[t]{2}{*}{0.1} & Present & 0.06158 & 0.16483 & 0.26024 & 1.01826 & 1.04543 & 1.84228 \\
\hline & & $\mathrm{AGH}$ & 0.06158 & 0.16484 & 0.26027 & 1.01826 & 1.04544 & 1.84229 \\
\hline & \multirow[t]{2}{*}{0.5} & Present & 0.32108 & 0.51064 & 0.84136 & 1.13768 & 1.34520 & 1.74345 \\
\hline & & $\mathrm{AGH}$ & 0.32101 & 0.51065 & 0.84138 & 1.13767 & 1.34521 & 1.74344 \\
\hline & \multirow[t]{2}{*}{1.0} & Present & 0.73322 & 1.00433 & 1.43616 & 1.43616 & 1.91681 & 2.00869 \\
\hline & & $\mathrm{AGH}$ & 0.78856 & 1.00483 & 1.41665 & 1.43618 & 1.91684 & 2.00872 \\
\hline \multirow[t]{8}{*}{2} & \multirow[t]{2}{*}{0.0} & Present & 0.03596 & 0.19158 & 0.35146 & 1.02229 & 1.08211 & 1.82135 \\
\hline & & $\mathrm{AGH}$ & 0.03596 & 0.19161 & 0.35149 & 1.02230 & 1.08213 & 1.82136 \\
\hline & \multirow[t]{2}{*}{0.1} & Present & 0.05456 & 0.22783 & 0.38247 & 1.02966 & 1.09493 & 1.81191 \\
\hline & & $\mathrm{AGH}$ & 0.05456 & 0.22784 & 0.38247 & 1.02966 & 1.09492 & 1.81191 \\
\hline & \multirow[t]{2}{*}{0.5} & Present & 0.33333 & 0.54008 & 0.87974 & 1.15062 & 1.37682 & 1.74257 \\
\hline & & $\mathrm{AGH}$ & 0.33332 & 0.54009 & 0.87975 & 1.15063 & 1.37683 & 1.74257 \\
\hline & \multirow[t]{2}{*}{1.0} & Present & 0.83333 & 1.01913 & 1.41767 & 1.45418 & 1.93217 & 2.02637 \\
\hline & & $\mathrm{AGH}$ & 0.80015 & 1.01911 & 1.41769 & 1.45421 & 1.93221 & 2.02639 \\
\hline \multirow[t]{8}{*}{3} & \multirow[t]{2}{*}{0.0} & Present & 0.09554 & 0.28718 & 0.49297 & 1.04542 & 1.15516 & 1.78653 \\
\hline & & AGH & 0.09556 & 0.28721 & 0.49302 & 1.04543 & 1.15517 & 1.78652 \\
\hline & \multirow[t]{2}{*}{0.1} & Present & 0.10639 & 0.30949 & 0.51769 & 1.05171 & 1.16737 & 1.78117 \\
\hline & & $\mathrm{AGH}$ & 0.10638 & 0.30951 & 0.51772 & 1.00517 & 1.16737 & 1.78116 \\
\hline & \multirow[t]{2}{*}{0.5} & Present & 0.35967 & 0.58365 & 0.93778 & 1.17221 & 1.42710 & 1.74382 \\
\hline & & $\mathrm{AGH}$ & 0.35967 & 0.58365 & 0.93779 & 1.17220 & 1.42709 & 1.74383 \\
\hline & \multirow[t]{2}{*}{1.0} & Present & 0.72932 & 1.04213 & 1.42532 & 1.47738 & 1.95646 & 2.05673 \\
\hline & & $\mathrm{AGH}$ & 0.81956 & 1.04226 & 1.42537 & 1.47741 & 1.95649 & 2.05677 \\
\hline \multirow[t]{8}{*}{4} & \multirow[t]{2}{*}{0.0} & Present & 0.16994 & 0.38254 & 0.63690 & 1.07704 & 1.24634 & 1.75990 \\
\hline & & $\mathrm{AGH}$ & 0.16997 & 0.38258 & 0.63697 & 1.07706 & 1.24635 & 1.75991 \\
\hline & 0.1 & Present & 0.17916 & 0.39824 & 0.65666 & 1.08278 & 1.25734 & 1.75718 \\
\hline & & $\mathrm{AGH}$ & 0.17917 & 0.39825 & 0.65666 & 1.08277 & 1.25739 & 1.75717 \\
\hline & 0.5 & Present & 0.40148 & 0.63760 & 1.00884 & 1.20232 & 1.49258 & 1.75090 \\
\hline & & $\mathrm{AGH}$ & 0.40149 & 0.63760 & 1.00895 & 1.20233 & 1.49260 & 1.75099 \\
\hline & 1.0 & Present & 0.88660 & 1.07349 & 1.43943 & 1.50490 & 1.98824 & 2.10042 \\
\hline & & $\mathrm{AGH}$ & 0.84673 & 1.07349 & 1.43945 & 1.50493 & 1.98827 & 2.10048 \\
\hline
\end{tabular}

AGH, Armenakis et al. (1969). 
Table 2. Comparison of Dimensionless Natural Frequencies for Two Elastic Layers

\begin{tabular}{clcccccc}
\hline & \multicolumn{7}{c}{ Dimensionless Natural Frequencies } \\
\cline { 2 - 7 }$n$ & & $\Omega_{1}$ & $\Omega_{2}$ & $\Omega_{3}$ & $\Omega_{4}$ & $\Omega_{5}$ & $\Omega_{6}$ \\
\hline 0 & Present & 0.13828 & 0.18946 & 1.02055 & 1.85681 & 2.03136 & 3.00075 \\
& AGH & 0.13829 & 0.18946 & 1.02055 & 1.85681 & 2.03137 & 3.00076 \\
1 & Present & 0.06158 & 0.16484 & 0.26027 & 1.01826 & 1.04544 & 1.84228 \\
& AGH & 0.06158 & 0.16484 & 0.26027 & 1.01826 & 1.04544 & 1.84229 \\
2 & Present & 0.05456 & 0.22784 & 0.38247 & 1.02966 & 1.09492 & 1.81191 \\
& AGH & 0.05456 & 0.22784 & 0.38247 & 1.02966 & 1.09492 & 1.81191 \\
3 & Present & 0.10638 & 0.30951 & 0.51772 & 1.05170 & 1.16736 & 1.78117 \\
& AGH & 0.10638 & 0.30951 & 0.51772 & 1.05170 & 1.16737 & 1.78116 \\
4 & Present & 0.17917 & 0.39825 & 0.65665 & 1.08277 & 1.25739 & 1.75717 \\
& AGH & 0.17917 & 0.39825 & 0.65666 & 1.08277 & 1.25739 & 1.75717 \\
\hline
\end{tabular}

$H / R=0.3, H / L=0.1, r_{0}=1.0$ in., $r_{1}=1.15$ in., and $r_{2}=1.3529$ in. AGH, Armenakis et al. (1969).

pared with those for an equivalent single-layer elastic cylinder. Results for two different geometries are given in Tables 2 and 3 . Table 2 presents the results for $H / R=0.3$ and $H / L=0.1$ for a laminated cylinder with radii of $r_{0}=1.0 \mathrm{in} ., r_{1}=$ $1.15 \mathrm{in}$., and $r_{2}=1.3529 \mathrm{in}$. This is equivalent to a single layer of $H / R=0.3$. Table 3 compares the dimensionless natural frequencies for two elastic laminated cylinders for $H / R=0.5$ and $H / L=$ 0.5 , and inner radii for layers are $r_{0}=1.0 \mathrm{in} ., r_{1}$ $=1.166 \overline{6}$ in., and $r_{2}=1.666 \overline{6}$ in. As indicated in these tables, computed results are very close to the results presented by Armenakas et al. (1969) for an equivalent single cylinder. Dimensionless natural frequencies for the multilayered cylinder are defined as $\Omega_{j}=\omega_{j} H /\left(\pi V_{21}\right)$, where $\omega_{j}$ is the $j$ th natural frequency and $V_{21}$ is the velocity of the shear wave for the first layer.

\section{Case 3: Three-Layer Elastic Cylinder}

Dimensionless natural frequencies for two different three-layer cylinders with the same elastic properties are compared with those of equivalent single cylinders, and are provided in Tables 4 and 5. Table 4 presents the results for $H / R=0.3$ and $H / L=0.1$ with radii of $r_{0}=1.0 \mathrm{in}$., $r_{1}=1.15 \mathrm{in}$., $r_{2}=1.2 \mathrm{in}$., and $r_{3}=1.3529 \mathrm{in}$. This is equivalent to a single layer of $H / R=0.3$. Table 5 compares the dimensionless natural frequencies for three layered cylinder with $H / R=0.5$ and $H / L=0.5$. The radii for layers are $r_{0}=1.0$ in., $r_{1}=1.166 \overline{6}$ in., $r_{2}=1.333 \overline{3}$ in., and $r_{3}=1.666 \overline{6}$ in. The two tables show the computed results are in excellent agreement with those reported by Armenakas et al. (1969) for an equivalent single cylinder.

\section{Case 4: Three-Layered Sandwiched Cylinder with Viscoelastic Core}

In this case a three-layer sandwich cylinder with a viscoelastic core is considered. All layers are assumed to have the same elastic properties. Energy dissipation in the core layer is allowed by introducing complex elastic moduli. The sandwiched cylinder has $H / R=0.3$ and $H / L=0.0$

Table 3. Comparison of Dimensionless Natural Frequencies for Two Elastic Layers

\begin{tabular}{|c|c|c|c|c|c|c|c|}
\hline \multirow[b]{2}{*}{$n$} & \multicolumn{7}{|c|}{ Dimensionless Natural Frequencies } \\
\hline & & $\Omega_{1}$ & $\Omega_{2}$ & $\Omega_{3}$ & $\Omega_{4}$ & $\Omega_{5}$ & $\Omega_{6}$ \\
\hline \multirow[t]{2}{*}{0} & Present & 0.36913 & 0.83335 & 1.34362 & 1.75354 & 2.34374 & 3.00328 \\
\hline & $\mathrm{AGH}$ & 0.36919 & 0.83334 & 1.34362 & 1.75354 & 2.34374 & 3.00328 \\
\hline \multirow[t]{2}{*}{1} & Present & 0.35885 & 0.53632 & 0.86722 & 1.17272 & 1.37979 & 1.75152 \\
\hline & $\mathrm{AGH}$ & 0.35884 & 0.53632 & 0.86723 & 1.17273 & 1.37980 & 1.75153 \\
\hline \multirow[t]{2}{*}{2} & Present & 0.37309 & 0.61399 & 0.95428 & 1.21002 & 1.47866 & 1.75287 \\
\hline & AGH & 0.37308 & 0.61400 & 0.95428 & 1.21002 & 1.47867 & 1.75288 \\
\hline \multirow[t]{2}{*}{3} & Present & 0.44122 & 0.71206 & 1.06769 & 1.27295 & 1.61076 & 1.78279 \\
\hline & $\mathrm{AGH}$ & 0.44121 & 0.71206 & 1.06769 & 1.27295 & 1.61076 & 1.78280 \\
\hline \multirow[t]{2}{*}{4} & Present & 0.55006 & 0.82384 & 1.18781 & 1.35816 & 1.71423 & 1.89819 \\
\hline & $\mathrm{AGH}$ & 0.55006 & 0.82384 & 1.18781 & 1.35817 & 1.71424 & 1.89820 \\
\hline
\end{tabular}

$H / R=0.5, H / L=0.5, r_{0}=1.0$ in., $r_{1}=1.166 \overline{6}$ in., and $r_{2}=1.666 \overline{6}$ in. AGH, Armenakis et al. (1969). 
Table 4. Comparison of Dimensionless Natural Frequencies for Three Elastic Layers

\begin{tabular}{llcccccc}
\hline & \multicolumn{7}{c}{ Dimensionless Natural Frequencies } \\
\cline { 2 - 7 }$n$ & & $\Omega_{1}$ & $\Omega_{2}$ & $\Omega_{3}$ & $\Omega_{4}$ & $\Omega_{5}$ & $\Omega_{6}$ \\
\hline 0 & Present & 0.13828 & 0.18946 & 1.02055 & 1.85681 & 2.03136 & 3.00075 \\
& AGH & 0.13829 & 0.18946 & 1.02055 & 1.85681 & 2.03137 & 3.00076 \\
1 & Present & 0.06158 & 0.16484 & 0.26027 & 1.01826 & 1.04544 & 1.84228 \\
& AGH & 0.06158 & 0.16484 & 0.26027 & 1.01826 & 1.04544 & 1.84229 \\
2 & Present & 0.05456 & 0.22784 & 0.38247 & 1.02966 & 1.09492 & 1.81191 \\
& AGH & 0.05456 & 0.22784 & 0.38247 & 1.02966 & 1.09492 & 1.81191 \\
3 & Present & 0.10638 & 0.30951 & 0.51772 & 1.05170 & 1.16736 & 1.78117 \\
& AGH & 0.10638 & 0.30951 & 0.51772 & 1.05170 & 1.16737 & 1.78116 \\
4 & Present & 0.17917 & 0.39825 & 0.65665 & 1.08277 & 1.25739 & 1.75717 \\
& AGH & 0.17917 & 0.39825 & 0.65666 & 1.08277 & 1.25739 & 1.75717 \\
\hline
\end{tabular}

$H / R=0.3, H / L=0.1, r_{0}=1.0$ in., $r_{1}=1.15$ in., $r_{2}=1.2$ in., and $r_{3}=1.35294$ in.

Table 5. Comparison of Dimensionless Natural Frequencies for Three Elastic Layers

\begin{tabular}{|c|c|c|c|c|c|c|c|}
\hline \multirow[b]{2}{*}{$n$} & \multicolumn{7}{|c|}{ Dimensionless Natural Frequencies } \\
\hline & & $\Omega_{1}$ & $\Omega_{2}$ & $\Omega_{3}$ & $\Omega_{4}$ & $\Omega_{5}$ & $\Omega_{6}$ \\
\hline \multirow[t]{2}{*}{0} & Present & 0.36918 & 0.83335 & 1.34362 & 1.75354 & 2.34374 & 3.00328 \\
\hline & AGH & 0.36919 & 0.83334 & 1.34362 & 1.75354 & 2.34374 & 3.00328 \\
\hline \multirow[t]{2}{*}{1} & Present & 0.35883 & 0.53632 & 0.86723 & 1.17272 & 1.37979 & 1.75152 \\
\hline & $\mathrm{AGH}$ & 0.35884 & 0.53632 & 0.86723 & 1.17273 & 1.37980 & 1.75153 \\
\hline \multirow[t]{2}{*}{2} & Present & 0.37308 & 0.61399 & 0.95428 & 1.21002 & 1.47866 & 1.75287 \\
\hline & $\mathrm{AGH}$ & 0.37308 & 0.61400 & 0.95428 & 1.21002 & 1.47867 & 1.75288 \\
\hline \multirow[t]{2}{*}{3} & Present & 0.44122 & 0.71206 & 1.06769 & 1.27295 & 1.61076 & 1.78279 \\
\hline & $\mathrm{AGH}$ & 0.44121 & 0.71206 & 1.06769 & 1.27295 & 1.61076 & 1.78280 \\
\hline \multirow[t]{2}{*}{4} & Present & 0.55006 & 0.82384 & 1.18781 & 1.35816 & 1.71423 & 1.89819 \\
\hline & $\mathrm{AGH}$ & 0.55006 & 0.82384 & 1.18781 & 1.35817 & 1.71424 & 1.89820 \\
\hline
\end{tabular}

$H / R=0.5, H / L=0.5, r_{0}=1.0$ in., $r_{1}=1.166 \overline{6}$ in., $r_{2}=1.333 \overline{3}$ in., and $r_{3}=1.666 \overline{6}$ in.

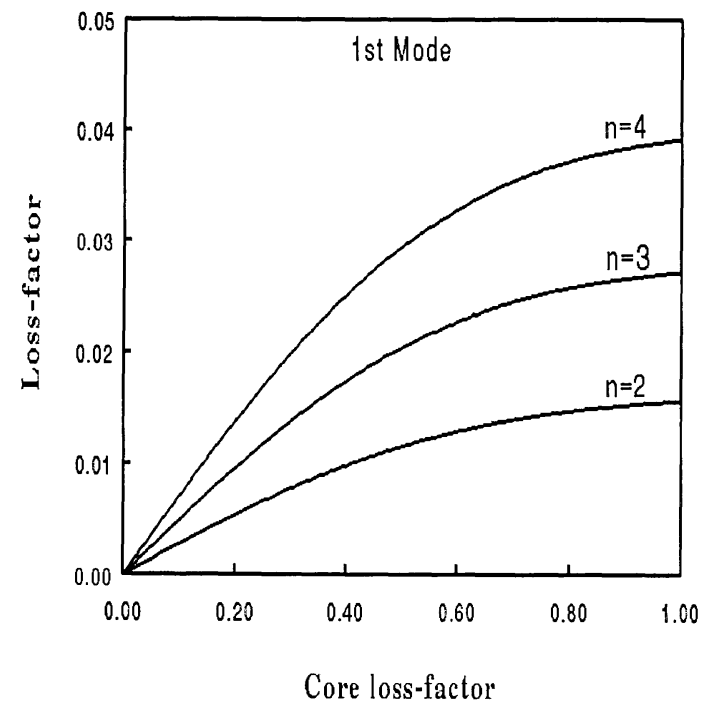

FIGURE 2 Variation of loss factor versus material loss factor of the core layer for the first mode.

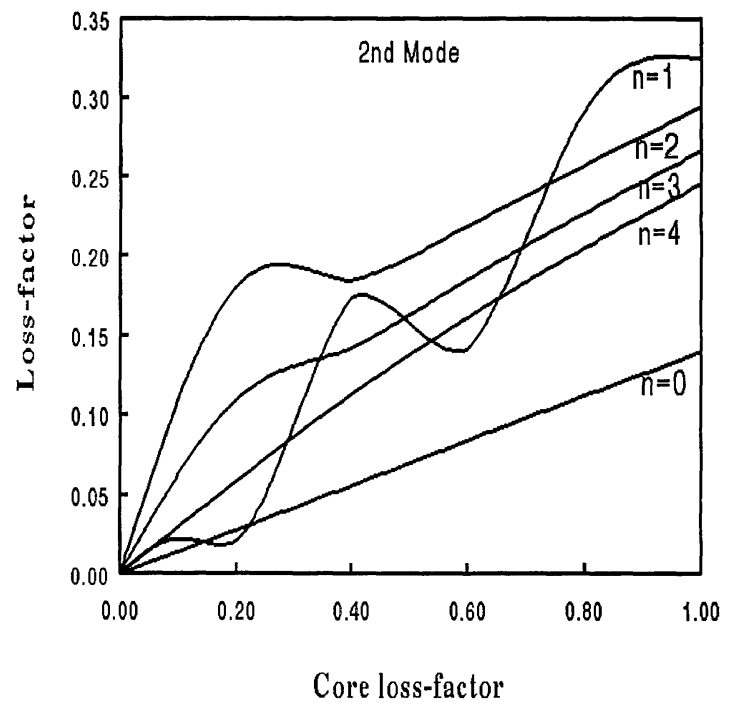

FIGURE 3 Variation of loss factor versus material loss factor of the core layer for the second mode. 
Table 6. Variation of Dimensionless Natural Frequencies $\left(\Omega_{j}\right)$ and Corresponding Loss-Factors $\left(\eta_{j}\right)$ with Respect to Core Material Loss-Factor $\left(\beta_{2}\right)$ for Different Circumferential Wave Numbers $(H / L=0.0)$

\begin{tabular}{|c|c|c|c|c|c|c|c|}
\hline \multirow[b]{2}{*}{$n$} & \multirow[b]{2}{*}{$\beta_{2}$} & \multicolumn{2}{|c|}{ Mode I } & \multicolumn{2}{|c|}{ Mode II } & \multicolumn{2}{|c|}{ Mode III } \\
\hline & & $\Omega_{1}$ & $\eta_{1}$ & $\Omega_{2}$ & $\eta_{2}$ & $\Omega_{3}$ & $\eta_{3}$ \\
\hline \multirow[t]{7}{*}{0} & 0.00 & 0.00000 & 0.00000 & 0.16306 & 0.00000 & 1.00345 & 0.00000 \\
\hline & 0.10 & 0.00000 & 0.00000 & 0.16306 & 0.01393 & 1.01818 & 0.02765 \\
\hline & 0.20 & 0.00000 & 0.00000 & 0.16306 & 0.02786 & 1.02134 & 0.05403 \\
\hline & 0.40 & 0.00000 & 0.00000 & 0.16307 & 0.05572 & 1.03291 & 0.09896 \\
\hline & 0.60 & 0.00000 & 0.00000 & 0.16307 & 0.08357 & 1.04912 & 0.13000 \\
\hline & 0.80 & 0.00000 & 0.00000 & 0.16307 & 0.11142 & 1.06695 & 0.14736 \\
\hline & 1.00 & 0.00000 & 0.00000 & 0.16307 & 0.13927 & 1.08416 & 0.15417 \\
\hline \multirow[t]{7}{*}{1} & 0.00 & 0.00000 & 0.00000 & 0.09584 & 0.00000 & 0.22524 & 0.00000 \\
\hline & 0.10 & 0.00000 & 0.00000 & 0.12366 & 0.02242 & 0.22524 & 0.01420 \\
\hline & 0.20 & 0.00000 & 0.00000 & 0.12315 & 0.02070 & 0.22524 & 0.02840 \\
\hline & 0.40 & 0.00000 & 0.00000 & 0.12117 & 0.17362 & 0.22525 & 0.05678 \\
\hline & 0.60 & 0.00000 & 0.00000 & 0.11819 & 0.14075 & 0.22527 & 0.08513 \\
\hline & 0.80 & 0.00000 & 0.00000 & 0.12676 & 0.29102 & 0.22528 & 0.11344 \\
\hline & 1.00 & 0.00000 & 0.00000 & 0.12706 & 0.32479 & 0.22531 & 0.14171 \\
\hline \multirow[t]{6}{*}{2} & 0.00 & 0.03597 & 0.00000 & 0.19161 & 0.00000 & 0.35149 & 0.00000 \\
\hline & 0.20 & 0.03598 & 0.00537 & 0.20881 & 0.18057 & 0.35151 & 0.02874 \\
\hline & 0.40 & 0.03603 & 0.00979 & 0.21200 & 0.18407 & 0.35156 & 0.05741 \\
\hline & 0.60 & 0.03608 & 0.01285 & 0.21572 & 0.21878 & 0.35163 & 0.08599 \\
\hline & 0.80 & 0.03614 & 0.01468 & 0.21768 & 0.25698 & 0.35172 & 0.11447 \\
\hline & 1.00 & 0.03619 & 0.01564 & 0.21843 & 0.29433 & 0.35182 & 0.14287 \\
\hline \multirow[t]{6}{*}{3} & 0.00 & 0.09556 & 0.00000 & 0.28721 & 0.00000 & 0.49302 & 0.00000 \\
\hline & 0.20 & 0.09564 & 0.00953 & 0.29555 & 0.10909 & 0.49307 & 0.02922 \\
\hline & 0.40 & 0.09585 & 0.01734 & 0.30136 & 0.14136 & 0.49321 & 0.05830 \\
\hline & 0.60 & 0.09612 & 0.02266 & 0.30584 & 0.18492 & 0.49343 & 0.08719 \\
\hline & 0.80 & 0.09640 & 0.02572 & 0.30812 & 0.22684 & 0.49370 & 0.11588 \\
\hline & 1.00 & 0.09665 & 0.02716 & 0.30893 & 0.26654 & 0.49401 & 0.14439 \\
\hline \multirow[t]{6}{*}{4} & 0.00 & 0.16997 & 0.00000 & 0.38258 & 0.00000 & 0.63696 & 0.00000 \\
\hline & 0.20 & 0.17018 & 0.01374 & 0.38636 & 0.05853 & 0.63708 & 0.02997 \\
\hline & 0.40 & 0.17071 & 0.02503 & 0.39263 & 0.11232 & 0.63741 & 0.05970 \\
\hline & 0.60 & 0.17141 & 0.03274 & 0.39696 & 0.16076 & 0.63791 & 0.08907 \\
\hline & 0.80 & 0.17213 & 0.03716 & 0.39906 & 0.20475 & 0.63853 & 0.11808 \\
\hline & 1.00 & 0.17278 & 0.03918 & 0.39960 & 0.24571 & 0.63925 & 0.14676 \\
\hline
\end{tabular}

with radii of $r_{0}=1.0$ in., $r_{1}=1.15$ in., $r_{2}=1.2$ in., and $r_{3}=1.3529$ in.

The effect of the core material loss factor on the dimensionless natural frequencies and their corresponding modal loss factors for circumferential wave numbers of $n=0,1,2,3$, and 4 are studied. The computed results for three consecutive modes are presented in Table 6. Figures 2 and 3 illustrate the variation of the loss factor for the first and the second modes versus material loss factor of the core layer for different circumferential wave numbers. In general, results indicate that for these modes higher material loss factor provide better damping capability for all modes except for the second mode with $n=1$. Three modes studied here are lowest extensional, breathing, and axial shear modes for $n=0$. For $n>0$, these modes are flexural (associated with large radial displacement), axial shear, and breathing. It should be noted that the given configuration is not designed for achieving maximum damping.

\section{CONCLUSION}

An analytical solution for the free vibration of thick viscoelastic multilayer cylinders is formulated. The developed method determines the natural frequencies and their corresponding modal loss factors for different circumferential wave number. In a special case of the three-layer sandwiched cylinder, the effect of different material damping for viscoelastic core constrained by two 
elastic layers is studied. Comparison of the dimensionless resonant frequencies for one-, two-, and three-layer elastic cylinders, of the same properties with a single layer equivalent cylinder, verified the validity of the analytical method presented.

\section{APPENDIX}

The following are the elements of the matrices $[C S]$ and $[S N]$. Only the nonzero elements are given.

$$
\begin{aligned}
& C S_{i j}=0, \quad \text { when } i \neq j \\
& C S_{11}=C S_{44}=\cos n \theta \cos (\omega t+\zeta z) \\
& C S_{22}=C S_{55}=\sin n \theta \cos (\omega t+\zeta z) \\
& C S_{33}=C S_{66}=\cos n \theta \sin (\omega t+\zeta z) \\
& S N_{11}=\alpha J_{n}^{\prime}(\alpha r), \quad S N_{12}=\alpha Y_{n}^{\prime}(\alpha r) \\
& S N_{13}=\zeta J_{n+1}(\beta r), \quad S N_{14}=\zeta Y_{n+1}(\beta r) \\
& S N_{15}=\frac{n}{r} J_{n}(\beta r), \quad S N_{16}=\frac{n}{r} Y_{n}(\beta r) \\
& S N_{21}=-\frac{n}{r} J_{n}(\alpha r), \quad S N_{22}=-\frac{n}{r} Y_{n}(\alpha r) \\
& S N_{23}=\zeta J_{n+1}(\beta r), \quad S N_{24}=\zeta Y_{n+1}(\beta r) \\
& S N_{25}=-\beta J_{n}^{\prime}(\beta r), \quad S N_{26}=-\beta Y_{n}^{\prime}(\alpha r) \\
& S N_{31}=-\zeta J_{n}(\alpha r), \quad S N_{32}=-\zeta Y_{n}(\alpha r) \\
& S N_{33}=-\beta J_{n+1}^{\prime}(\beta r)-\frac{n+1}{r} J_{n+1}(\beta r) \\
& S N_{34}=-\beta Y_{n+1}^{\prime}(\beta r)-\frac{n+1}{r} Y_{n+1}(\beta r) \\
& S N_{41}=-\lambda\left(\alpha^{2}+\zeta^{2}\right) J_{n}(\alpha r)+2 \mu \alpha^{2} J_{n}^{\prime \prime}(\alpha r) \\
& S N_{42}=-\lambda\left(\alpha^{2}+\zeta^{2}\right) Y_{n}(\alpha r)+2 \mu \alpha^{2} Y_{n}^{\prime \prime}(\alpha r) \\
& S N_{43}=2 \mu \zeta \beta J_{n+1}^{\prime}(\beta r), \quad S N_{44}=-2 \mu \zeta \beta Y_{n+1}^{\prime}(\beta r) \\
& S N_{45}=2 \mu \frac{n}{r} \beta J_{n}^{\prime}(\beta r)-2 \mu \frac{n}{r^{2}} J_{n}(\beta r) \\
& S N_{46}=2 \mu \frac{n}{r} \beta Y_{n}^{\prime}(\beta r)-2 \mu \frac{n}{r^{2}} Y_{n}(\beta r) \\
& S N_{51}=-2 \mu \frac{n}{r} \alpha J_{n}^{\prime}(\alpha r)+2 \mu \frac{n}{r^{2}} J_{n}(\alpha r) \\
& S(\alpha)
\end{aligned}
$$

$$
\begin{aligned}
S N_{52}= & -2 \mu \frac{n}{r} \alpha Y_{n}^{\prime}(\alpha r)+2 \mu \frac{n}{r^{2}} Y_{n}(\alpha r) \\
S N_{53}= & -\mu \zeta \frac{n+1}{r} J_{n+1}(\beta r)+\mu \zeta \beta J_{n+1}^{\prime}(\beta r) \\
S N_{54}= & -\mu \zeta \frac{n+1}{r} Y_{n+1}(\beta r)+\mu \zeta \beta Y_{n+1}^{\prime}(\beta r) \\
S N_{55}= & -\mu \frac{n^{2}}{r^{2}} J_{n}(\beta r)-\mu \beta^{2} J_{n}^{\prime \prime}(\beta r)+\frac{\mu}{r} \beta J_{n}^{\prime}(\beta r) \\
S N_{56}= & -\mu \frac{n^{2}}{r^{2}} Y_{n}(\beta r)-\mu \beta^{2} Y_{n}^{\prime \prime}(\beta r)+\frac{\mu}{r} \beta Y_{n}^{\prime}(\beta r) \\
S N_{61}= & -2 \mu \zeta \alpha J_{n}^{\prime}(\alpha r), \quad S N_{62}=-2 \mu \zeta \alpha Y_{n}^{\prime}(\alpha r) \\
S N_{63}= & \mu\left(\frac{n+1}{r^{2}}-\zeta^{2}\right) \mathrm{J}_{n+1}(\beta r) \\
& -\mu\left(\frac{n+1}{r}\right) \beta J_{n+1}^{\prime}(\beta r)-\mu \beta^{2} J_{n+1}^{\prime \prime}(\beta r) \\
S N_{64}= & \mu\left(\frac{n+1}{r^{2}}-\zeta^{2}\right) Y_{n+1}(\beta r) \\
& -\mu\left(\frac{n+1}{r}\right) \beta Y_{n+1}^{\prime}(\beta r)-\mu \beta^{2} Y_{n+1}^{\prime \prime}(\beta r) \\
S N_{65}= & -\mu \zeta \frac{n}{r} J_{n}(\beta \mathbf{r}), \quad S N_{66}=-\mu \zeta \frac{n}{r} Y_{n}(\beta r)
\end{aligned}
$$

where prime denotes differentiation with respect to the arguments of the Bessel function.

\section{REFERENCES}

Armenakas, A. E., 1967, "Propagation of Harmonic Waves in Composite Circular Cylindrical Shells. Part I: Theoretical Investigation,' AIAA Journal, Vol. 5 , pp. 740-744.

Armenakas, A. E., 1971, "Propagation of Harmonic Waves in Composite Circular Cylindrical Shells. Part II: Numerical Results,' AIAA Journal, Vol. 9, pp. 599-605.

Armenakas, A. E., Gaxis, D. C., and Hermann, G., 1969, Free Vibrations of Circular Cylindrical Shells, Pergamon Press, Oxford.

Braga, A. M., Barbone, P. E., and Herman, G., 1990, "Wave Propagation in Fluid-Loaded Laminated Cylindrical Shells," Proceedings of U.S. Congress of Applied Mechanics, Vol. 43, No. 5, pp. S359-S365.

Hamidzadeh, H. R., and Chandler, D. E., 1991, "Circumferential Vibrations of Three Layered Sandwich Cylinders," Proceedings of the Biennial ASME Con- 
ference on Mechanical Vibrations and Noise, Vol. DE-36, pp. 233-237.

Huang, K. H., and Dong, S. B., 1984, "Propagation Waves and Edge Vibrations in Anisotropic Composite Cylinders," Journal of Sound and Vibration, Vol. 96, pp. 363-379.

Rattanawangcharoen, N., and Shah, A. H., 1991, "Vi- bration of Laminated Isotropic Cylinders," Proceedings of the Canadian Congress of Applied Mechanics, pp. 230-231.

Rattanawangcharoen, N., Shah, A. H., and Datta, S. K., 1992, "Wave Propagation in Laminated Composite Circular Cylinders," International Journal of Solids Structures, Vol. 29, No. 6, pp. 767-781. 

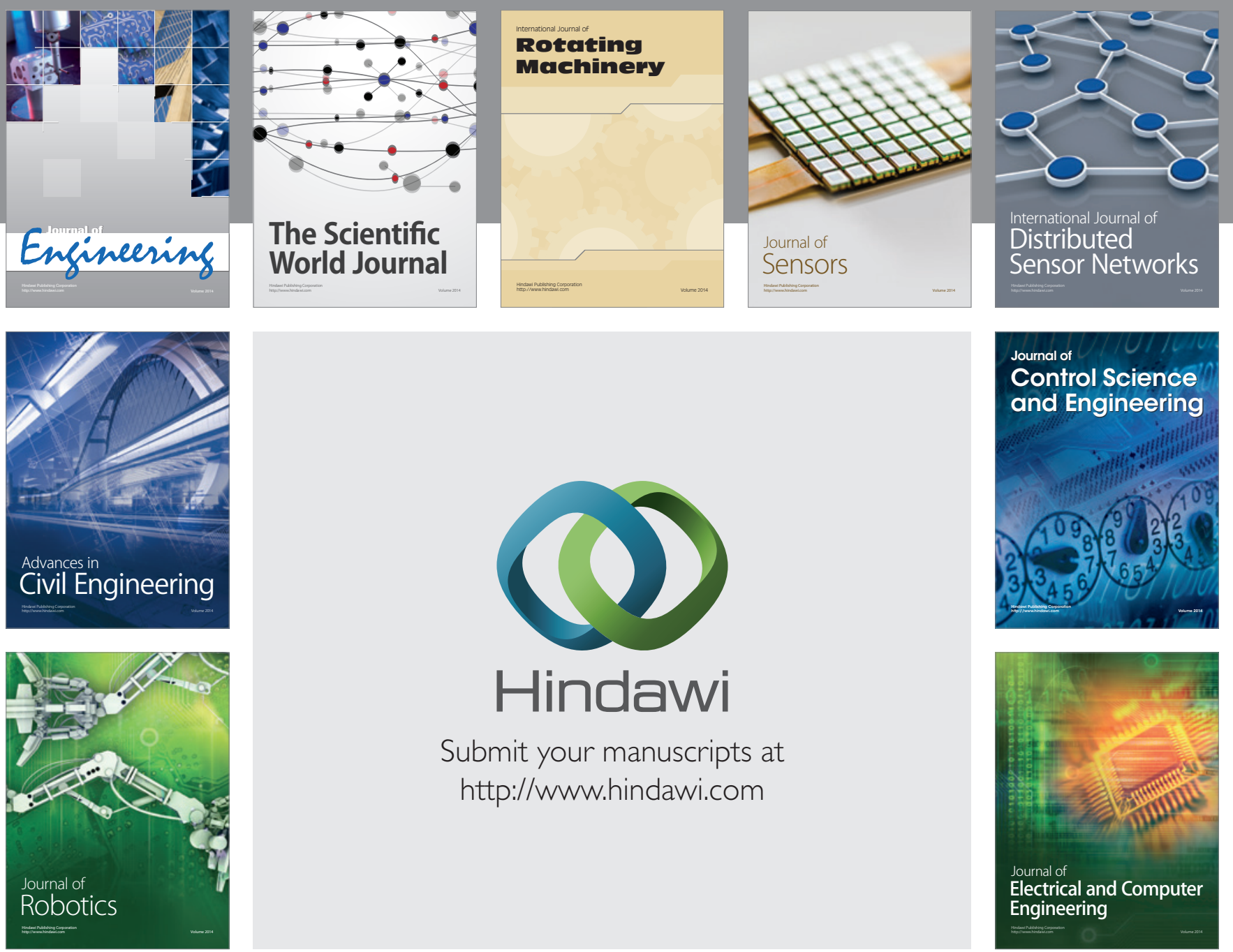

Submit your manuscripts at

http://www.hindawi.com
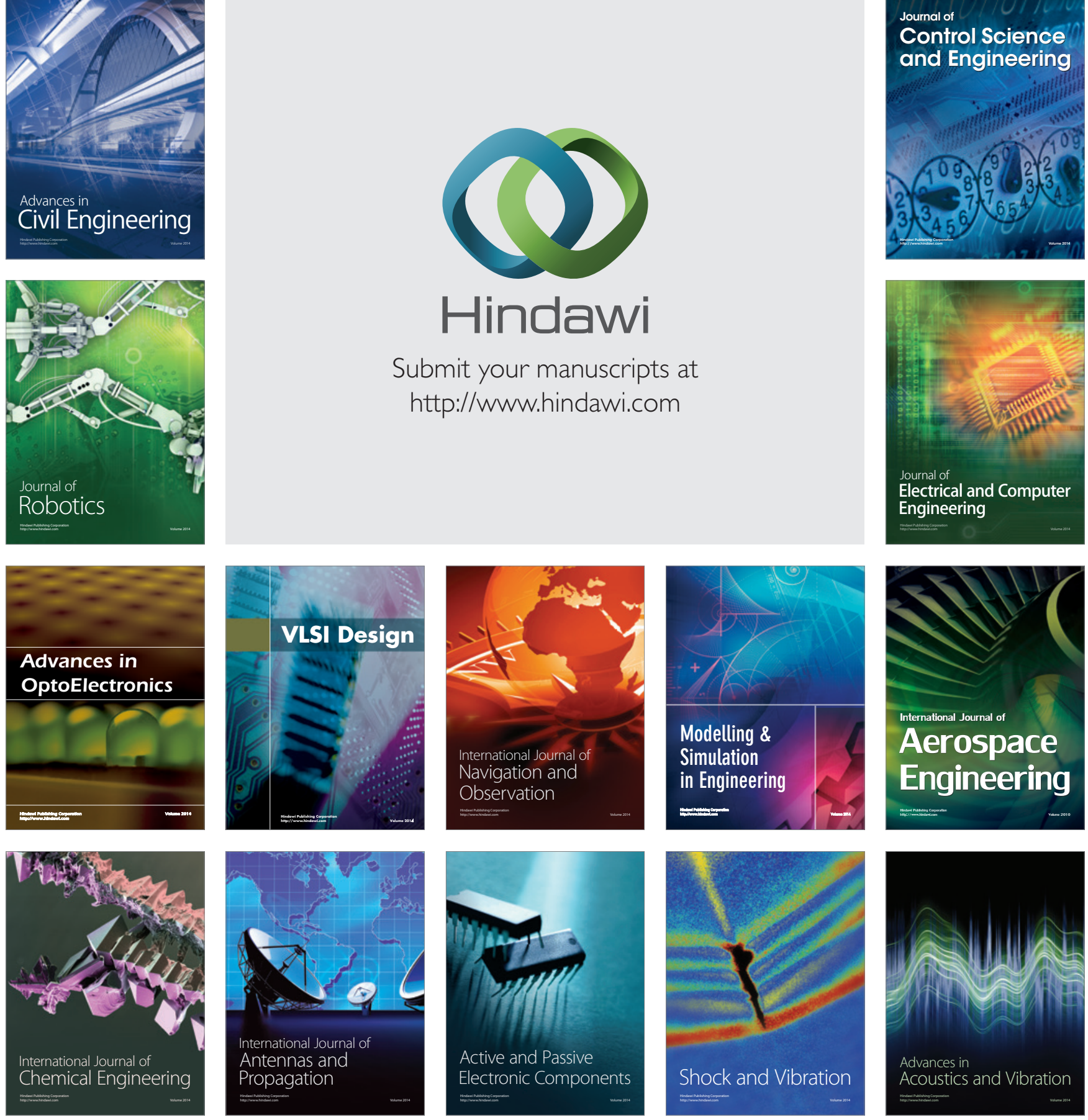